\title{
Guest editorial: special section on software quality assurance and quality management
}

\author{
Dietmar Winkler $\cdot$ Stefan Biffl
}

Published online: 17 June 2014

(C) Springer Science+Business Media New York 2014

This special section comprises research papers and industry experience papers which are grouped by the theme of Quality Assurance and Quality Management.

The first two papers in this section handle different aspects of risk management: risk management in the medical domain and the application of static quality assurance approaches in small and medium enterprises for risk mitigation, respectively.

1. Christin Lindholm, Jesper Pedersen Notander, and Martin Höst. "A Case Study on Software Risk Analysis and Planning in Medical Device Development"

This research paper reports on a case study in medical device development organizations to investigate risk management processes. Experiences from risk management method applications have been systematically captured and analyzed with action research. The analysis of risk management processes focused on risk identification, risk analysis, and risk planning and identified challenging problems in the risk management process with respect to the definition of the system boundary and system context, the use of scenarios as input to the risk identification, estimation of detectability during risk analysis, and action proposals during risk planning. The authors conclude that the risk management method has the potential to be used in development organizations to improve risk management processes.

2. Mario Gleirscher, Dmitriy Golubitskiy, Maximilian Irlbeck, and Stefan Wagner: "Introduction of Static Quality Analysis in Small and Medium-Sized Software Enterprises: Experience from Technology Transfer" (industry experience paper)

D. Winkler $(\bowtie) \cdot$ S. Biffl

Christian Doppler Laboratory for Software Engineering Integration for Flexible Automation Systems (CDL-Flex), Institute of Software Technology and Interactive Systems, Vienna University of Technology, Favoritenstrasse 9/188, 1040 Vienna, Austria e-mail: dietmar.winkler@tuwien.ac.at

S. Biffl

e-mail: stefan.biffl@tuwien.ac.at 
This industrial experience paper presents a case study that investigates the application of three automated static quality assurance approaches (clone detection, bug pattern detection, and architecture conformance analysis) in five collaborating SME organizations. The application of static quality assurance activities can help in mitigating risks and increasing software quality. The authors analyzed the effects of applying automated quality assurance approaches by using the quality model Quamoco, an approach to support quality modeling and evaluation, and concluded that the application of automated static quality assurance approaches is valuable for SMEs to mitigate risk and improve software product quality.

The third paper in this special section is focused on a strategy for establishing risk-based testing in industrial test processes.

3. Michael Felderer and Rudolf Ramler: "Integrating Risk-Based Testing in Industrial Test Processes"

This industry experience paper reports the implementation of a risk-based testing process in an industrial environment. Risk-based testing helps to optimize resource allocation and provides decision support for managers to plan testing. The authors provide a process approach to establish risk-based testing in existing test processes in an organization and present the evaluation results of a pilot application. They report on their experiences, and insights with a focus on the benefits, prerequisites, and challenges that they had to face during the implementation. The benefits of risk-based testing include faster detection of defects and more reliable quality statements for each release.

Acknowledgments We would like to thank the Editor-in-Chief of the Software Quality Journal, Rachel Harrison, for encouraging us to assemble this special issue. Of course, this special issue would not have been possible without the outstanding work by the many reviewers involved in selecting the papers. 